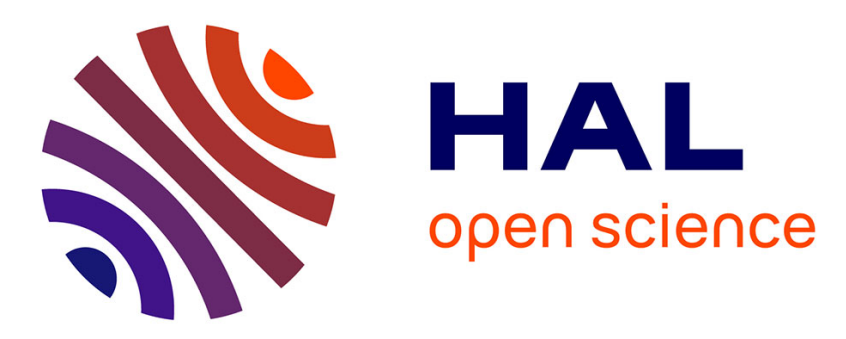

\title{
Anxiété et coping des soignants : la prison est-elle un facteur de protection?
}

A.H. Boudoukha, M. Hautekeete, D. Hautekeete-Sence, V. Cousin, F. Calesse

\section{To cite this version:}

A.H. Boudoukha, M. Hautekeete, D. Hautekeete-Sence, V. Cousin, F. Calesse. Anxiété et coping des soignants : la prison est-elle un facteur de protection ?. Annales Médico-Psychologiques, Revue Psychiatrique, 2009, 167 (8), pp.583. 10.1016/j.amp.2009.03.013 . hal-00580181

\section{HAL Id: hal-00580181 \\ https://hal.science/hal-00580181}

Submitted on 27 Mar 2011

HAL is a multi-disciplinary open access archive for the deposit and dissemination of scientific research documents, whether they are published or not. The documents may come from teaching and research institutions in France or abroad, or from public or private research centers.
L'archive ouverte pluridisciplinaire HAL, est destinée au dépôt et à la diffusion de documents scientifiques de niveau recherche, publiés ou non, émanant des établissements d'enseignement et de recherche français ou étrangers, des laboratoires publics ou privés. 


\section{Accepted Manuscript}

Title: Anxiété et coping des soignants : la prison est-elle un facteur de protection?

Authors: A.H. Boudoukha, M. Hautekeete, D.

Hautekeete-Sence, V. Cousin, F. Calesse

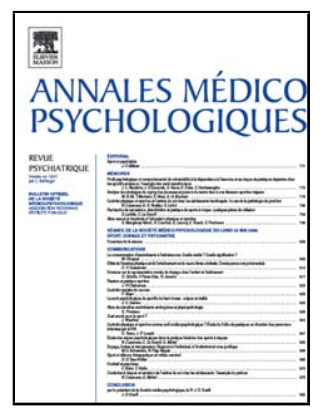

PII: $\quad$ S0003-4487(09)00127-9

DOI: $\quad$ doi:10.1016/j.amp.2009.03.013

Reference: $\quad$ AMEPSY 1013

To appear in:

Received date: $\quad 11-6-2008$

Accepted date: $\quad$ 20-3-2009

Please cite this article as: Boudoukha AH, Hautekeete M, Hautekeete-Sence D, Cousin $\mathrm{V}$, Calesse F, Anxiété et coping des soignants: la prison est-elle un facteur de protection ?, Annales medio-psychologiques (2008), doi:10.1016/j.amp.2009.03.013

This is a PDF file of an unedited manuscript that has been accepted for publication. As a service to our customers we are providing this early version of the manuscript. The manuscript will undergo copyediting, typesetting, and review of the resulting proof before it is published in its final form. Please note that during the production process errors may be discovered which could affect the content, and all legal disclaimers that apply to the journal pertain. 


\section{Mémoire}

\section{Anxiété et coping des soignants : la prison est-elle un facteur de protection ?} Anxiety and coping of nursing staff: Is prison a factor of protection

\section{A. H. Boudoukha ${ }^{\text {a }}$, M. Hautekeete ${ }^{\text {b }}$, D. Hautekeete-Sence ${ }^{\text {b }}$, V. Cousin ${ }^{c}$, F. Calesse ${ }^{\text {c }}$}

a) Université de Nantes, UFR de Psychologie, Laboratoire de Psychologie «Éducation, Cognition \& Développement », EA 3259

b) Université Lille 3 Charles-de-Gaulle - UFR de Psychologie- UPRES TEC, EA 2453

c) Service Médico-Psychologique Régional - Maison d'arrêt de Loos-les-Lille.

Auteur correspondant : Abdel Halim Boudoukha, Université de Nantes, UFR de Psychologie, Laboratoire de Psychologie « Education, Cognition \& Développement », EA 3259, Nantes, France

Adresse mail : Abdel-halim.boudoukha@univ-nantes.fr

\section{Résumé}

Stress et anxiété sont des problèmes récurrents dans le développement humain. Certaines situations les exacerbent alors qu'inversement, certains mécanismes tel le coping les modèrent. Nous étudions cette question par le biais d'une recherche sur l'anxiété et les copings des soignants en milieu carcéral. Nous posons comme hypothèses que ces sujets rapporteront une anxiété-état importante en raison de la nature imprévisible du contexte carcéral. Nous pensons également qu'ils rapporteront des copings orientés vers l'émotion comme conséquence de cette anxiété-état. Dans un premier temps, nous avons recueilli les sources de stress et les copings de 13 soignants en prison lors d'un entretien. Nous avons ensuite créé une échelle de coping $a d$ hoc. Dans un deuxième temps, nous avons proposé à 50 soignants auprès de personnes détenues le questionnaire d'anxiété-état STAI-Y ainsi que notre échelle de coping. Contrairement à nos hypothèses, nous observons des niveaux d'anxiété-état faibles. Ils ne se distinguent pas selon des variables démographiques (sexe, âge, ancienneté, profession) pas plus que selon des variables pénitentiaires (temps d'implication auprès des détenus ou du type de prisons). Les copings sont centrés sur le problème, contrairement à notre hypothèse. Nous ne notons pas de différence dans la fréquence de leur utilisation en fonction des variables démographiques. En revanche, le fait de travailler en maison d'arrêt ou quotidiennement auprès de détenus augmente la fréquence d'utilisation de copings centrés sur le problème. Nous discutons ces surprenants résultats à l'aune de la 
théorie transactionnelle-cognitive, de la perception de la prison (imprévisibilité vs contrôlabilité) et de la représentation cognitive des personnes incarcérées (patients vs détenus).

\section{Abstract}

Anxiety and stress are current and real human issues. These may worsen in certain situations whereas mechanisms known as coping mediate the effect of stress and anxiety, state-anxiety more specifically. The aim of this study concerns this issue, via a research on state-anxiety and coping among nursing staff in French prisons. The nursing staff of prisons was chosen for two major reasons: First, nursing is typically described as a stressful occupation, consequently stress, coping and anxiety have been widely studied in this population. Second, prisons are generally considered as "tough" places in which the inmates are perceived as "dangerous". Indeed, assaults occur frequently in prison. Therefore prison nursing staff should in all probability report stress and anxiety and the ways in which they try to cope with them. Consequently, the author assumed that state-anxiety levels should be high for nursing staff due to the unpredictable prison setting. The author also assumed that this staff should report emotion-focused coping instead of problem-focused coping because of the high levels of state-anxiety.

The research comprised two steps. The first one was an exploratory study comprising interviews of 13 members of the nursing staff (physicians, nurses, caregivers, etc.) working in a French prison in the north of France. The author reviewed all stressful events and how the staff coped with them. Then a coping inventory was designed on the basis of the "Way-ofCoping Check-List". This was accomplished by a re-transcription of the coping cases collected during the interviews. The second study was experimental. Fifty other members of the nursing staff working in other prisons in the north of France were involved. They were asked to fill out the "state form" of the STAI-Y and the Coping Inventory. The effects of demographic and "prison-inmate" variables on state-anxiety and coping were analysed.

The results do not support the author's assumptions. First of all, state-anxiety levels remained low in the sample. There were no significant differences between the subjects' stateanxiety levels according to demographic and prison-inmate variables. Second, contrary to the assumptions, nursing staff used problem-focused coping more frequently than emotionfocused coping. Coping frequencies used did not differ according to the demographic variables but according to the prison-inmate variables. In fact, nursing staff in jail more frequently use problem-focused coping than prison nursing staff: In fact, permanent prison 
nursing staff use problem-focused coping more frequently than part-time nursing staff . Moreover, nursing staff who work with inmates every day use problem-focused coping more frequently than nursing staff who work with inmates part-time. The author discusses and explains the results on the basis of the cognitive-transactional theory of stress and coping, and of anxiety and the cognitive construction of danger (cognitive evaluation of the prison and the inmates). The results are likely to have implications on psychotherapy and staff training.

Mots clés : Anxiété ; Coping; Détenus ; Prison ; Soignants ; Stress

Keywords: Anxiety; Cognitive Evaluation; Coping; Inmates; Medical Staff; Nurse; Prison; Stress

Les soins proposés aux personnes incarcérées en France sont assurés par les services relevant de l'hôpital ${ }^{1}$. Le personnel soignant hospitalier, qui forme désormais un «tiers » dans la relation autrefois exclusive entre personnel pénitentiaire et personnes incarcérées, a requis des efforts d'adaptation $[15,42]$. Les soins en milieu carcéral sont en effet, plus complexes qu'ils ne le sont en milieu hospitalier en raison du contexte et de la particularité des patients que sont les personnes détenues [2]. En effet, les relations difficiles avec ces patients ont des répercussions non seulement au niveau des prises en charge mais également sur la santé des professionnels, notamment en termes de stress, d'anxiété ou de burnout [5,27,31]. La prison s'avère donc un lieu propice à leur étude, puisqu'elle réunit un ensemble de facteurs qui y concourent, et également, par voie de conséquence, à l'étude des stratégies par lesquelles le personnel s'y ajuste, ou coping.

\section{Stress, anxiété et coping chez les soignants}

L'apparition d'un sentiment de fatigue, d'insomnies ou de plaintes relatives aux comportements des patients fait partie des symptômes rapportés par les soignants [49]. Le stress et l'anxiété sont souvent évoqués pour rendre compte de leur souffrance [39].

En ce qui concerne le stress, on peut le considérer comme une «transaction entre la personne et l'environnement que le sujet évalue comme débordant ses ressources et qui compromet son bien-être » [35]. Il est la résultante de deux processus que sont les évaluations

\footnotetext{
${ }^{1}$ Unité de Consultations et de soins Ambulatoires (UCSA) et Service Médico-Psychologique Régional (SMPR).
} 
cognitives et le coping ${ }^{2}$. Ce dernier a des implications prépondérantes dans la façon dont on s'ajuste au stress puisqu'il réfère à l'ensemble des efforts cognitifs et comportementaux de gestion des situations évaluées comme menaçantes ou débordant nos ressources [35]. Il s'agit d'un processus temporel, dynamique et conscient et non d'une caractéristique stable ou un trait de personnalité. Certains auteurs admettent néanmoins l'idée de coping-trait ou de style de coping pour décrire certains copings dont l'usage est prépondérant chez un sujet [8]. Quelle qu'en soit sa description, le coping a des implications en terme de gestion des situations avec les copings dit «centrés sur le problème» et en termes de régulation des émotions avec les copings dits « centrés sur l'émotion » [34, 35].

Par ailleurs, une importante conséquence de la perception ou de l'évaluation de situations menaçantes s'exprime par une augmentation transitoire de l'anxiété, plus précisément de l'anxiété-état [26]. Il s'agit de réactions émotionnelles qui se caractérisent par des sentiments de tension, de nervosité, d'inquiétude et par l'activation ou l'éveil du système autonome. Ces états sont souvent transitoires mais peuvent réapparaître sous l'effet de stimuli appropriés [46].

Stress, anxiété-état et coping sont donc des phénomènes dynamiques et inter-reliés qui ont une importance sur la santé [1,26]. Ils sont également dépendants du contexte et certaines situations sont plus susceptibles de donner lieu à leur étude. C'est le cas par exemple de la prison.

\section{La prison : un cumul de facteurs de stress chez les professionnels?}

Les études montrent de manière consistante la prison comme un cumul de facteurs de stress chez les professionnels, en raison notamment de la nature stressante, anxiogène, voire traumatique des relations avec les personnes détenues [3,7,20,22,38]. En effet, les personnes incarcérées représentent une population très particulière. Leur nombre ne cesse de croître depuis de nombreuses années. Selon les chiffres de l'administration pénitentiaire, on compte au début de l'année 2008 plus de 65842 personnes incarcérées, alors qu'il y en avait 49000 en 2001 [21]. En conséquence, les demandes de consultation affluent alors que les capacités des personnels soignants pour y répondre restent les mêmes, générant un état de frustration chez les personnes incarcérées [7].

Par ailleurs, si l'état de santé général des détenus est jugé satisfaisant par les services de soins en milieu carcéral, c'est sur le plan de la santé mentale que l'on observe des

\footnotetext{
${ }^{2}$ Les termes de « stratégies d'ajustement » ou « stratégies de faire face » sont parfois utilisés pour traduire « coping ».
} 
pathologies mentales de plus en plus sévères $[19,14]$. En effet, les recherches montrent que les personnes incarcérées en France sont particulièrement touchées par les pathologies psychiques que l'on retrouve particulièrement dans des tableaux cliniques comorbides, qu'il s'agisse de troubles de l'humeur, de troubles psychotiques, de troubles anxieux ou encore de troubles addictifs. La prévalence de ces troubles psychiques est, de plus, très élevée : plus de $60 \%$ pour les troubles thymiques et plus de $42 \%$ pour les troubles psychotiques $[23,40,41]$.

Enfin, découlant directement de l'augmentation de la population carcérale et de la présence de plus en plus importante de troubles mentaux sévères chez les personnes incarcérées, on remarque une augmentation des agressions des personnels [7,11,30,43]. Si les personnels soignants sont moins touchés par ces événements traumatiques, il n'en demeure pas moins qu'ils exercent dans un milieu typiquement défini comme dangereux, dont la probabilité d'incidents est plus élevée qu'en milieu hospitalier [44,48].

Nous pouvons, sans conteste, affirmer que la prison réunit un ensemble de facteurs qui concourent au stress et à l'anxiété en face desquels les soignants mettent en œuvre des copings. Il n'existe pourtant pas de travaux sur cette problématique chez les soignants en prison en France. Cette recherche exploratoire aura donc comme objectif d'appréhender cette problématique par le biais de l'analyse de l'anxiété-état et des copings mobilisés par les personnels soignant en prison.

\section{Hypothèses}

$\mathrm{Au}$ regard de notre revue de littérature, nous pensons que les personnels soignants travaillant en milieu carcéral devraient présenter des niveaux d'anxiété-état importants en raison de la nature peu contrôlable voire imprévisible de l'environnement carcéral. Ces niveaux devraient être plus importants chez ceux qui travaillent en maison d'arrêt, en raison de la surpopulation carcérale, par rapport à ceux qui exercent en centre de détention, où le nombre de détenus n'excède pas le nombre de place. En ce qui concerne les copings, nous pensons qu'en lien avec des niveaux d'anxiété-état importants, les copings des soignants devraient refléter des copings plus « centrés sur l'émotion » que « centrés sur le problème ». 


\section{Méthode}

\subsection{Recherche clinique qualitative}

\subsubsection{Les entretiens exploratoires}

Nous avons mené des entretiens exploratoires afin d'évaluer les événements stressants et les copings. Nous avons pour cela rencontré 13 soignants (trois médecins, trois psychologues, quatre infirmières et trois assistantes sociales) dans un service de soins en maison d'arrêt durant un entretien semi-directif d'une demi-heure chacun. Ces sujets ont participé volontairement à la recherche, sans rétribution.

Une consigne orale et une grille d'entretien ont été rédigées sous forme écrite afin d'assurer un maximum de standardisation. L'entretien était cependant conduit sous forme de discussion afin d'offrir une liberté de parole aux sujets.

Cet entretien permettait de recueillir les sources de stress et ensuite de discuter des copings. Deux domaines ont donc été explorés au cours des entretiens :

- Les événements stressants de leur quotidien de travail en prison. Nous leur posions la question suivante: Quels sont les événements qui vous stressent dans votre travail?

- Les copings. Nous leur demandions : Que faites-vous pour «faire face » à ces événements stressants?

Ce deuxième domaine exploré nous a permis de créer une échelle de coping ad hoc.

\subsubsection{La création d'une échelle de coping}

Le choix de la création d'un outil ad hoc, c'est-à-dire contextuel, pour mesurer les stratégies de coping plutôt que l'utilisation d'une échelle existante est justifié par trois raisons principales. Premièrement, le coping, dans l'approche cognitive transactionnelle, est considéré comme un processus dynamique et dépendant des stresseurs et des individus [36]. Par ailleurs, les différents questionnaires de type générique présentent des mesures du coping considéré comme un processus ou comme un trait. En conséquence, la comparaison des différentes études entre elles est complexe, d'autant que les situations stressantes sont très variables dans celles qui utilisent des outils spécifiques. Cette absence d'homogénéité les rend difficilement utilisables per se dans une recherche [18]. Enfin, à une seule exception, la CISS, les propriétés psychométriques des différents questionnaires sont souvent faibles [25]. 
Pour les besoins de la création de notre échelle de coping, nous avons retranscrit littéralement chaque entretien sur informatique. Nous nous sommes inspirés de la «Way of coping check-list » de Lazarus et Folkman [35], validée en France [16; 32] et de ces huit souséchelles de coping que sont :

- la résolution de problème ;

- l'acceptation de confrontation ;

- la prise de distance ;

- la réévaluation positive ;

- l'auto-accusation ;

- la fuite-évitement;

- la recherche d'un soutien social

- et enfin la maîtrise de soi.

Toutes les stratégies de coping retranscrites à partir des entretiens ont été recodées selon la méthode des juges selon les huit sous-échelles de coping. La méthode des juges, comme son nom l'indique, réunit des personnes considérées comme spécialiste d'un domaine [12]. Dans le cadre de notre création d'une échelle de coping, 25 "juges » en psychologie (professeur, maîtres de conférences, doctorants et étudiants d'un niveau au moins égal à bac +4$)$ devaient décider de la pertinence de la stratégie de coping dans l'une des huit souséchelles. Nous avons retenu cinq items pour chaque sous-catégorie pour deux raisons : a) Ils étaient les plus spécifiques d'une catégorie de coping donnée et b) nous souhaitions élaborer un outil qui ne contienne pas un nombre trop important d'items, pour éviter les effets liés à la fatigue.

L'ordre des items a été organisé aléatoirement afin que les items appartenant à chaque sous-échelle de coping ne se succèdent pas pour éviter les effets d'amorçage. L'échelle de coping contient donc 40 items.

\section{Recherche expérimentale quantitative}

\subsection{Population}

Il s'agit de 50 sujets travaillant auprès de personnes incarcérées dans des services de soin en maison d'arrêt ou en centre de détention dans le nord de la France. On compte 38 femmes et 12 hommes pour une moyenne d'âge de 37,2 ans (écart-type $=9,3$ ) et d'ancienneté de 4,6 ans (écart type $=3,3$ ans). Ces sujets sont tous volontaires et participe à la recherche sans rétribution. 


\subsection{Matériel}

\subsubsection{La forme française du State and trait Anxiety Inventory de Spielberger (STAI-Y)}

Le STAI-Y contient 40 items qui mesurent l'anxiété d'un sujet. Il est composé de deux sous-échelles indépendantes. Nous avons choisi la forme française Y-A du questionnaire c'est-à-dire celle qui mesure l'anxiété-état $[9,46]$. La STAY-Y a été largement utilisée dans la recherche en psychologie et notamment pour étudier les effets de l'anxiété sur le stress psychologique. La sensibilité de l'échelle anxiété-état dans le stress situationnel a également été démontrée à plusieurs reprises [9].

La forme Y-A se compose de 20 items que le sujet évalue lui-même. Il doit les coter au fur et à mesure, sans en oublier un seul, sur une échelle Likert en quatre points (non ; plutôt non ; plutôt oui ; oui). La note totale pour un sujet peut varier de 20 à 80 .

\subsubsection{Le questionnaire de coping des soignants en milieu carcéral}

Ce questionnaire évalue les copings à travers huit sous-échelles qui contiennent chacune cinq items représentatifs de la catégorie (par exemple : «je ne réponds pas aux provocations » traduit un coping de maîtrise de soi ; "Je discute avec l'équipe, le personnel » illustre un coping de soutien social ; «j'ai essayé de ne pas penser aux problèmes » reflète un coping de fuite-évitement.)

Cet outil est composé de 40 items, il n'est donc pas trop long pour éviter les réponses stéréotypées et les items sont facilement compréhensibles. La cotation se fait sur une échelle de type Likert en 5 points (jamais ; rarement; parfois ; souvent ; presque toujours). Le sujet peut donc décrire au mieux la fréquence et le type des comportements ou pensées qu'il utilise pour « faire face » aux événements stressants.

\subsection{Procédure}

Après avoir obtenu l'accord conjoint des médecins chefs de Services MédicoPsychologiques Régional (SMPR) ou d’Unités de Consultations et de Soins Ambulatoires (UCSA) et des directeurs des établissements pénitentiaires, nous avons distribué le matériel de notre étude. Il s'agit d'un dossier composé de quatre parties. La première contient la consigne écrite qui décrit le cadre et les objectifs de la recherche. La deuxième est constituée de la forme Y-A de la STAI. La troisième est composée du questionnaire de Coping. Enfin, la dernière partie comporte les questions factuelles qui renseignent sur les caractéristiques descriptives de la population. 
Deux cas de figure sont à noter concernant la passation des questionnaires. Lorsque nous avions accès à l'établissement pénitentiaire, chaque sujet était sollicité individuellement, une présentation de l'étude avait lieu, en suivant la trame de la consigne écrite. Il leur était demandé de remplir les questionnaires en suivant l'ordre de présentation. Nous précisions que tous les résultats obtenus seraient confidentiels et qu'aucune donnée individuelle n'apparaîtrait.

Lorsque nous n'avions pas accès à l'établissement pénitentiaire, des photocopies des dossiers étaient envoyées dans les prisons à l'attention du service médical. Nous demandions au secrétariat de les mettre à disposition des soignants. Une consigne explicative écrite les aidait à remplir les questionnaires et à les renvoyer à l'adresse de l'université de rattachement des chercheurs.

\subsection{Opérationnalisation de nos variables}

En ce qui concerne les variables indépendantes (VI), nous avons distingué les sujets selon des facteurs démographiques et pénitentiaires.

Les variables démographiques regroupent le sexe, l'ancienneté, l'âge et la profession. Nous avons catégorisé l'ancienneté et recatégorisé la variable " profession » en deux niveaux, personnel médical et psychologues d'une part et personnels paramédicaux d'autre part. Ces catégorisations ont été créées afin de mener des traitements statistiques sur des groupes homogènes dont l'effectif est supérieur à dix sujets.

Les variables «pénitentiaires » sont des variables qui reflètent des éléments inhérents à la relation avec les personnes détenues. Parmi celles-ci, il y a « le temps d'implication avec les personnes incarcérées » avec deux degrés (temps plein vs temps partiel) et le type de « structure pénitentiaire » (Maison d'Arrêt vs Centre de Détention).

S'agissant de nos variables dépendantes (VD), nous disposons de neuf mesures qui sont le score d'anxiété-état et les scores aux huit catégories de coping.

\section{Résultats}

Les analyses statistiques ont été réalisées au moyen du logiciel StatView ${ }^{\odot}$ pour Windows version 5.0 (1992-1998). Pour l'ensemble des traitements statistiques, nous avons fixé le seuil de probabilité à 0.05 en deçà duquel nous rejetons l'hypothèse nulle et considérons l'effet observé comme significatif.

Pour les besoins de notre étude, nous avons procédé à des analyses de variances (ANOVA) afin de comparer les moyennes des catégories de coping. À la suite de celles-ci, 
nous avons choisi le test a posteriori de Bonferroni/Dunn, parce qu'il permet d'éliminer le risque d'erreur en cas de comparaisons multiples [33].

\subsection{L'anxiété-état}

La moyenne d'anxiété-état dans notre échantillon est de $33.1(\sigma=8.1)$. Les scores obtenus pour chaque niveau de chaque VI sont indiqués dans le tableau 1.

\section{Ici le tableau $n^{\circ} 1$}

Aucune variable indépendante (sexe, âge, ancienneté, profession, temps d'implication auprès des personnes incarcérées, type de structure pénitentiaire) n'a d'effet significatif sur l'anxiété-état (voir Tableau 2).

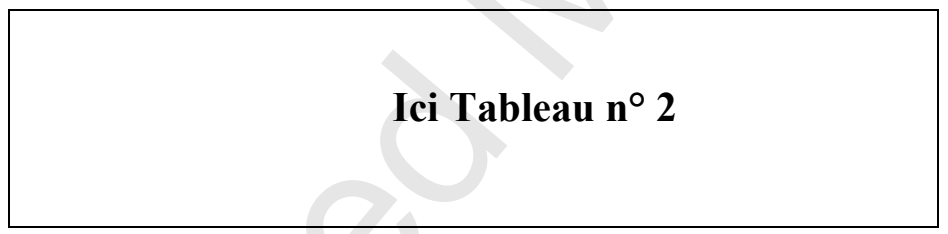

\subsection{Les copings.}

L'analyse de variance met en évidence une différence significative entre les fréquences d'utilisation des copings $\left(F_{(7,49)}=7.93 ; p<.001\right)$. Nous présentons les moyennes et les écarts-type de chaque catégorie de coping dans le tableau $n^{\circ} 3$ et la figure $n^{\circ} 1$.

\section{Ici Tableau $\mathbf{n}^{\circ} 3$}

Les tests a posteriori montrent que les copings de «fuite-évitement » et de «prise de distance » sont significativement moins utilisés que les copings de « résolution de problème », de « réévaluation positive », de « recherche de soutien social» et de «maîtrise de soi ». Le coping de «maîtrise de soi » est également plus préconisé que celui «d'acceptation de confrontation $»\left(\mathrm{cf}\right.$. Tableau $\left.\mathrm{n}^{\circ} 4\right)$. 


\section{Ici Figure 1}

\section{Ici tableau 4}

Par ailleurs, nous avons étudié l'impact de nos variables démographique et pénitentiaires sur les copings. Nous n'observons pas de différences significatives dans la fréquence d'utilisation des copings en fonction du sexe, de l'âge, de l'ancienneté ou de la profession. Nos variables démographiques n'ont donc pas d'impact significatif sur la fréquence d'utilisation des copings.

En ce qui concerne les variables pénitentiaires, certaines stratégies de coping se distinguent selon le temps d'implication avec les détenus. Ainsi, la résolution de problème et la recherche de soutien social sont significativement plus utilisées chez les sujets qui travaillent quotidiennement avec des personnes incarcérées, en comparaison de leurs homologues qui ne travaillent pas exclusivement auprès de personnes détenues (respectivement $t_{47}=2,693$ et $\mathrm{p}=.009 ; t_{47}=2,761$ et $\mathrm{p}=.008$ ). Enfin, la résolution de problème est significativement plus utilisée chez les sujets qui travaillent en maison d'arrêt, en comparaison de leurs homologues en centre pénitentiaire $\left(t_{47}=2,023\right.$ et $\left.\mathrm{p}=.048\right)$

\section{Discussion}

\subsection{Les soignants en prison : moins touchés ou mieux prémunis contre les événements menaçants?}

Un ensemble de résultats attirent d'emblée notre attention. Il s'agit de l'absence d'effets significatifs de nos variables démographiques et pénitentiaires sur les niveaux d'anxiété-état des soignants, ce qui infirme totalement nos hypothèses.

En ce qui concerne les niveaux d'anxiété-état des hommes et des femmes, l'absence de différence significative est pour le moins surprenante. En effet, la majorité des recherches portant sur la question montre, de manière solide et régulière, que les femmes rapportent des 
niveaux d'anxiété-état significativement plus intenses que ceux des hommes, en réaction aux mêmes événements. Ces résultats sont classiquement interprétés par le fait que les femmes expriment plus facilement et plus volontiers leurs émotions que les hommes, en raison des stéréotypes associés au sexe notamment [17,45].

Par ailleurs, nos variables pénitentiaires ne génèrent pas de différences significatives entre les niveaux d'anxiété-état de nos sujets. Ces résultats nous amènent, à l'instar de ceux des variables démographiques, à proposer trois hypothèses explicatives :

1. L'anxiété-état est médiatisée par des cognitions qui en modulent l'expression et l'intensité [24]. C'est le cas par exemple pour les troubles anxieux [4]. On pourrait donc penser que la prison, univers perçu de «l'extérieur » comme dangereux et menaçant, amenant des cognitions amorçant l'activation des processus d'anticipation anxieuse, pourrait s'avérer moins anxiogène, vécu de «l'intérieur ».

2. Il existe un lien important entre l'anxiété-état et l'anxiété-trait : plus l'anxiété-trait est importante, plus l'anxiété-état est élevée dans des situations stressantes [17,24,26,46]. Nous n'avons pas étudié l'anxiété-trait de nos sujets mais nous pourrions avancer l'idée que les soignants qui choisissent de travailler en prison sont moins enclins à ressentir ou exprimer une anxiété ou une appréhension anxieuse à la suite de situations menaçantes.

3. En lien direct avec la définition du stress dans l'approche cognitivo-transactionnelle [35,36] et de ses liens avec l'anxiété-état dans le modèle d'Endler [26], on peut poser l'hypothèse que les professionnels mettraient en place des copings leur permettant de gérer efficacement les situations stressantes voire anxiogènes. Nous reviendrons sur cette troisième explication dans le point consacré au coping.

Il nous semble cependant important de revenir sur le présupposé fort de cette recherche : le personnel soignant travaille en prison. Est-ce réellement le cas ? Cette question peut sembler absurde (il exerce dans une structure pénitentiaire) mais conduit à une interrogation sur la construction cognitive de la prison chez ce personnel qui dévoile une particularité : les personnes incarcérées sont, dans les services de soin, des patients. En effet, dans le cadre des structures soignantes en prison, les détenus sont, comme dans n'importe quel autre hôpital, considérés comme des patients. Nous pensons que cette perception des détenus en tant que patient, a) modifie totalement le contexte structurel : ce n'est plus la prison mais l'hôpital et b) propose un autre mode de relation : la relation soignant/soigné en place de la relation surveillant/surveillé. En conséquence, l'agressivité, la violence ou la frustration sont généralement peu exprimées envers les soignants en prison comme elles peuvent l'être envers les surveillants dans la mesure où les premiers proposent aux personnes 
incarcérées un espace de soins et non de contention. Par ailleurs, les passages à l'acte violent de certains patients, par exemple psychotiques, sont bien mieux évalués et pris en charge par les soignants qui sont, de par leur formation, mieux préparés. En conséquence, la partie hospitalière de la prison n'est probablement pas perçue comme un environnement menaçant ou dangereux et donc anxiogène. Cette hypothèse pourrait expliquer des niveaux d'anxiétéétat faibles.

\subsection{Les copings des soignants sont-ils efficaces?}

La fréquence d'utilisation des copings n'est pas uniforme chez nos sujets. Les copings que l'on peut ranger sous le vocable de «copings d'évitement ou centrés sur l'émotion » comme la fuite, l'évitement ou encore la prise de distance, sont significativement moins utilisés que les «copings de confrontation ou centrés sur le problème » comme la résolution de problème, la réévaluation positive ou encore la maîtrise de soi [35,36]. Cet ensemble de résultats infirme l'hypothèse formulée sur le coping, mais permet d'envisager des explications alternatives.

Par exemple, cette utilisation différenciée des copings ne semble pas liée à des caractéristiques démographiques dans notre recherche, mais plutôt à des caractéristiques pénitentiaires. En effet, nous observons une utilisation plus fréquente de copings centrés sur le problème par rapport aux copings centrés sur l'émotion, uniquement chez les soignants qui soit travaillent quotidiennement auprès de patients détenus ou soit exercent en maison d'arrêt. Les copings plus fréquemment utilisés nous amènent à penser que les soignants dans ces deux situations tentent de garder une distance vis-à-vis des personnes incarcérées et de conserver un sentiment de contrôle. S'agit-il d'un maintien de contrôle dans un univers qui en définitive n'est pas considéré comme incontrôlable?

Cette prédominance d'une utilisation de copings s'exprimant par une maîtrise de soi ou un contrôle dans un environnement typiquement décrit comme incontrôlable [37] nous y fait penser, mais peut sembler à la fois cohérent et contradictoire. La contradiction concerne les liens entre copings et événements stressants, notamment l'observation que lorsque des sujets évaluent une situation comme incontrôlable ou inchangeable, ils tendent à rapporter une prédominance de copings d'évitement ou centrés sur l'émotion. A contrario, si la situation est évaluée comme modifiable ou contrôlable, les copings centrés sur le problème ou de confrontation sont privilégiés $[10,28,47,50]$. Si l'on validait l'hypothèse "d'incontrôlabilité » de la prison, on aurait observé une fréquence plus élevée de l'utilisation de copings centrés sur l'émotion dans notre échantillon. La préconisation privilégiée de copings de maîtrise ou 
de contrôle indique le contraire, ce qui est cohérent en raison de la nature des détenus qui sont avant tout des patients. Les soignants adoptent donc des copings qui leur permettent de contrôler les événements qui pourraient être menaçants. En conséquence, pouvons-nous considérer les copings centrés sur le problème comme un facteur de protection?

Nos résultats sont cohérents avec les travaux relatifs aux liens entre santé et copings qui révèlent, de façon consistante, que les copings centrés sur l'émotion ou de fuite-évitement sont associés à un stress plus important que les copings orientés vers le problème ou l'action $[17,28,45,47,50]$. Ce type de résultat n'est pas récent. Il y a plus de 20 ans, une méta-analyse des études sur le coping chez des personnes malades montrait l'effet adaptatif à court terme des copings d'évitement et l'effet adaptatif à long terme des copings de confrontation à la maladie [47]. On observe donc une association de l'action de copings comme la résolution de problème avec le ralentissement de la progression de certaines maladies ou de certains symptômes, là encore, à l'inverse des copings d'évitement [13,36]. Il faut bien entendu mesurer ces résultats et ne pas établir de généralisation abusive en oubliant les caractéristiques individuelles et situationnelles qui doivent toujours être prises en compte [29].

\section{Conclusion}

En prison, les soignants font l'expérience de réalités différentes (le soin vs la peine), de structures distinctes (l'hôpital vs la prison) et d'une éthique différente (déontologie des soins/éthique de la justice). On pourrait penser que le milieu menaçant ou dangereux dans lequel ils vont exercer générerait une anxiété transitoire importante. Notre recherche infirme cette hypothèse chez les personnels soignant en prison. Il semble donc qu'ils aient mis en place des copings efficaces leur permettant de gérer les situations stressantes voire anxiogènes. La fréquence de copings centrés sur le problème comme la maitrise de soi ou la résolution de problème nous permet de penser que cette hypothèse est cohérente. La place qu'occupent les soignants en prison et les liens qu'ils nouent avec les personnes incarcérées méritent cependant des investigations futures. De même, des recherches comparant les copings des soignants exerçant à l'hôpital et en prison apporteront des indications intéressantes quant à l'efficacité de certains copings et seront utiles sur un plan psychothérapeutique dans le sens d'une démarche en psychologie positive [6].

Conflit d'intérêt : aucun 


\section{Références}

[1] Aldwin CM, Yancoura AY. Coping and health: A comparison of the stress and trauma literatures. In : Schnurr PP, Green BL. Physical Health Consequences of Exposure to Extreme Stress. Washington, DC: American Psychological Association; 2003.

[2] Archer E. Obligation de soin et secret médical. Une exigence éthique essentielle : la réflexion sur les fondements. In: $\mathrm{AFC}$, editor. XXXII ${ }^{\mathrm{e}}$ Congrès de Criminologie : Les soins obligés ou l'utopie de la triple entente; Lille: Dalloz; 2002. p. 163-7.

[3] Augestad LB, Levander S. Personality health and job stress among employees in a Norvegian penitentiary and in a maximum security hospital. Work and Stress. 1992;6:65-79.

[4] Beck AT, Emery G. Anxiety disorders and phobias: A cognitive perspective. New York: BasicBooks/HarperCollins Publishers; 1985.

[5] Bennet L, Kelaher M, Ross M. Quality of life in health care professionals: Burnout and its associated factors in HIV/AIDS related care. Psychology and Health 1994;9:273-83.

[6] Boudoukha AH, Courty B. Burnout des soignants: une perspective étiologique. $35^{\mathrm{e}}$ journée scientifique de l'association française de thérapie comportementale et cognitive. Paris, décembre 2007.

[7] Boudoukha AH. Étude conjointe du burnout et des troubles de stress traumatique dans une population à risques. Cas des professionnels en milieu carcéral. Villeneuve d'Ascq: Université Lille3 Charles-De-Gaulle; 2006.

[8] Bruchon-Schweitzer ML, Dantzer R. Introduction à la psychologie de la santé. Paris: PUF; 2003.

[9] Bruchon-Schweitzer ML, Paulhan I. Le Manuel du STAI-Y de CD Spielberger, adaptation française. Paris: ECPA; 1993.

[10] Busko V. Transactional stress and coping theory in accounting for psychological states measures. Medicinski glasnik. 2007;4:63-70.

[11] Casadamont G. Violence en détention. Paris: Direction de l'Administration Pénitentiaire; 2001.

[12] Caverni C. La verbalisation comme source d'observables pour l'étude du fonctionnement cognitif. In: Caverni J-P, Bastien C, Mendelsohn P, Tiberghien G, editors. Modèles et méthodes. Paris: PUF; 1988. p. 253-73.

[13] Chang AML, Bidewell JW, Huntington AD, Daly J, Johnson A, Wilson H, et al. A survey of role stress, coping and health in Australian and New Zealand Hospital nurses. International Journal of Nursing Studies. 2007;44:1354-62. 
[14] Coldefy M, Faure P, Prieto N. La santé mentale et le suivi psychiatrique des détenus. Études et Résultats. 2001;181:1-12.

[15] Colin M, Jean JP. Droit aux soins et amélioration de la condition des détenus : deux objectifs indissociables. Revue française des Affaires Sociales. 1997;1:17-29.

[16] Cousson F, Bruchon-Schweitzer M, Quintard B, Nuissier J, Rascle N. Analyse multidimentionnelle d'une échelle de coping : Validation française de la W.C.C. (Way of Coping Check List). Psychologie Française 1996;41-2:155-64.

[17] Cousson-Gélie F, Taylard A, Quintard B. L'évaluation des stratégies de coping chez les patients asthmatiques. Revue Européenne de Psychologie Appliquée 1998;48:89-94.

[18] Coyne JC, Racioppo MW. Never the twain shall meet? Closing the gap between coping research and clinical intervention research. American Psychologist 2000; 55:655-64.

[19] De Beaurepaire C. Psychopathologie et détention: données et réflexions cliniques. Revue française des Affaires Sociales 1997;1:213-24.

[20] De Conninck G, \& Loodts, Patrick. Les risques professionnels des surveillants de prison : recherche exploratoire sur le risque d'être agressé par les détenus et le syndrome de stress post-traumatique. Revue internationale de criminologie et de police technique et scientifique 1999;1:81-98.

[21] Direction de l'Administration Pénitentiaire. Statistiques mensuelles de la population écrouée et détenue en France. Paris: Ministère de la Justice; 2008.

[22] Dowden C, Tellier C. Predicting work-related stress in correctional officers: A metaanalysis. Journal of Criminal Justice 2004;32:31-47.

[23] Duburcq A, Bonte J, Coulomb S, Marchand C, Fagniani F, Falissard B. Étude épidémiologique sur la santé mentale des personnes détenues : rapport final. Paris: Ministère de la Santé - Ministère de la Justice; 2006.

[24] Endler NS, Kocovski NL. State and trait anxiety revised. Journal of anxiety disorders 2001;15:231-45.

[25] Endler NS, parker JD. Multidimensional assessment of coping: A critical evaluation. Journal of personnality and Social Psychology 1990;58:844-54.

[26] Endler NS. Stress, Anxiety and Coping: The multidimensional interaction model. Canadian Psychology / Psychologie Canadienne 1997;38:137-53.

[27] Florio GA, Donnelly JP, Zevon MA. The structure of work related stress and coping among oncology nurses in High-stress medical settings: A transactional analysis. J Occup Health Psychol 1998;3:227-42. 
[28] Folkman S, Lazarus, RS. An analysis of coping in a middle-aged community sample. Journal of health and social behavior 1980;21:219-39.

[29] Folkman S. Coping and health. In: Halonen S.F. Davis. The many faces of psychological research in the 21st century. Syracuse: Society for the Teaching of Psychology; 2001. P.14587.

[30] Galindo C. De la violence carcérale: Sources, Perceptions et modes d'expression [Doctorat]. Metz: Université de Metz; 2002.

[31] Garman AN, Corrigan PW, Morris S. Staff burnout and patient satisfaction: Evidence of relationships at the care unit level. Journal of Occupational Health Psychology. 2002;7:23541.

[32] Graziani PL, Rusinek S, Servant D, Hautekèete-Sence D, Hautekèete M. Validation française du questionnaire de coping "Way of coping check-list-revised" (W.C.C.-R.) et analyse des évenements stressants du quotidien. Journal de Thérapie Comportementale et Cognitive 1998;8:100-12.

[33] Howell DC. Méthodes statistiques en sciences humaines. Paris: De Boek; 1998.

[34] John OP, Gross JJ. Healthy and Unhealthy Emotion Regulation: Personality Processes, Individual Differences, and Life Span Development. Journal of Personality 2004;72:1301-34.

[35] Lazarus RS, Folkman S. Stress, Appraisal and Coping. New York: Springer; 1984.

[36] Lazarus RS. From psychological stress to the emotions: A history of changing outlooks. Annual Review of Psychology 1993;44:1-21.

[37] Lhuilier D, Aymard N. L'univers pénitentiaire : du côté des surveillants de prison. Paris: Desclée De Brouwer; 1997.

[38] Lourel M, Gana K, Prud'homme V, Cerclé A. le burn-out chez les personnels des maisons d'arrêt: test du modèle «demande-contrôle» de Karasek. L'Encéphale 2004;XXX:557-63.

[39] Marchand A, Demers A, Durand P. Does work really cause distress? The contribution of occupational structure and work organization to the experience of psychological distress. Social Science \& Medecine 2005;61:1-14.

[40] Mouquet MC, Dumont M, Bonnevie MC. La santé à l'entrée en prison : un cumul de facteurs de risque. Études et Résultats 1999;4:1-10.

[41] Mouquet MC. La santé des personnes entrées en prison en 2003. Études et Résultats. 2005:1-12.

[42] Rousseau E. La réforme de l'organisation des soins aux personnes détenues : les textes et leur mise en œuvre. Revue française des Affaires Sociales 1997;1:73-80. 
[43] Safran DA, Tartaglini AJ. Workplace violence in an urban jail setting. In: VandenBos GR, Bulatao EQ, editors. Violence of the job: Identifying risks and developing solutions. Washington DC: American Psychological Association; 1996. p. 207-16.

[44] Schat ACH, Kelloway KE. Reducing the adverse consequences of workplace aggression and violence: The buffering effects of organizational support. Journal of Occupational Health Psychology 2003; 8:110-22.

[[45]] Schultz SM, Alpers GW, Hofmann SG. Negative self focused cognitions mediate the effect of trait social anxiety on state anxiety. Behaviour Research and Therapy 2008;46:43849.

[[46]] Spielberger CD, Gorsuch RL, Lushene RE, Vaag PR, Jacobs GA. Manuel for the state anxiety inventory (STAI). Palo Alto: Consulting Psychologist Press; 1983.

[47] Suls J, Fletcher B. The relative efficacity of avoidant and non-avoidant coping strategies. Health Psychology 1985;4:249-88.

[48] Tartaro C. The impact of density on jail violence. Journal of Criminal Justice 2002;30:499-510.

[49] Van Dierendonck D, Schaufeli WB, Buunk BP. Burnout and inequity among human service professionals: A longitudinal study. Journal of Occupational Health Psychology 2001;6:43-52.

[50] Zeidner, M. Anxiety and coping with community disasters: the Israeli experience. Journal of research in personality 2007;41:213-20. 
Tableau 1 : Moyennes et écart-type d'anxiété-état en fonction des nos variables.

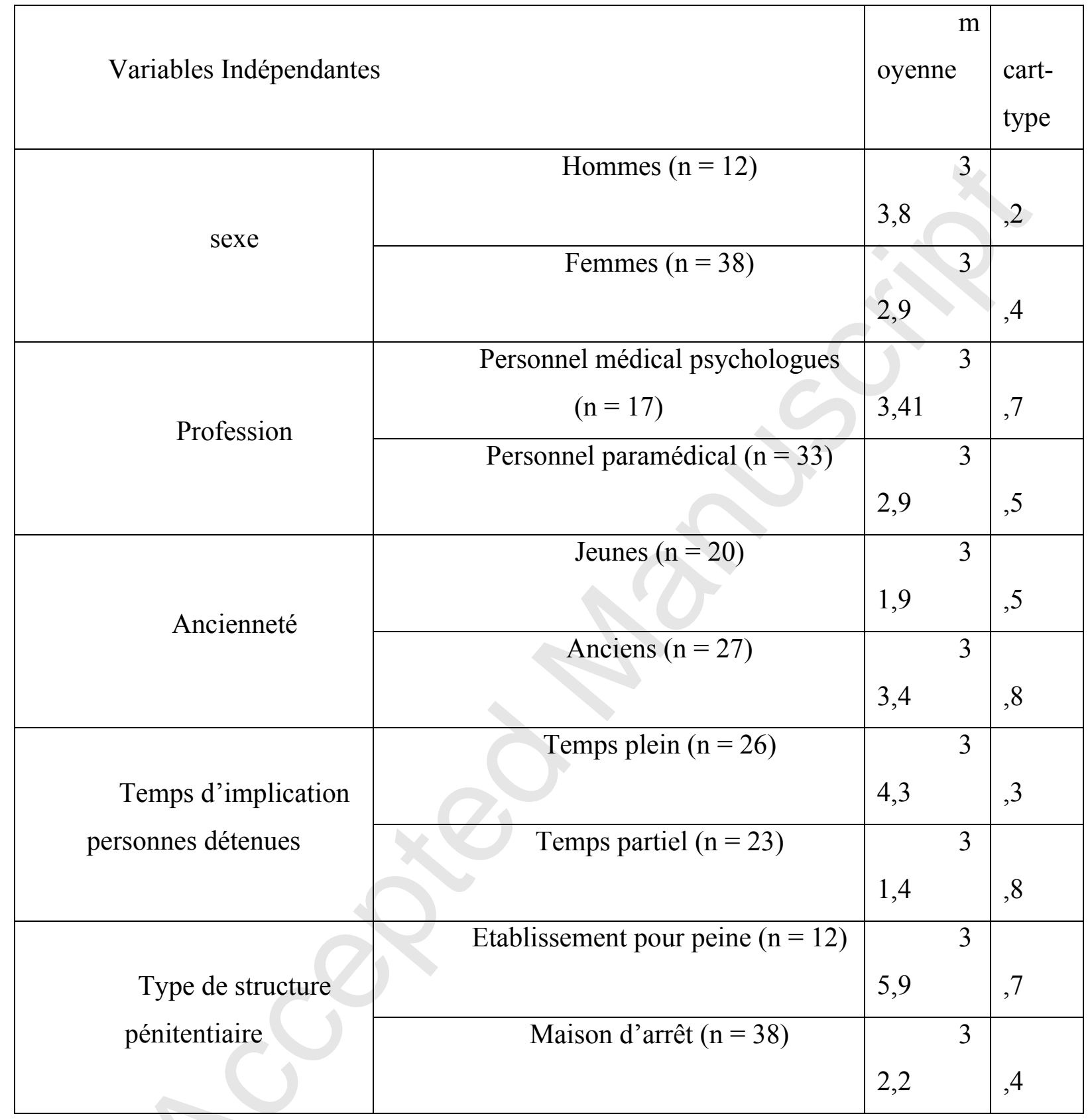


Tableau $\mathrm{n}^{\circ} 2$ : Niveaux d'anxiété-état en fonction de nos variables.

\begin{tabular}{|c|c|c|}
\hline Effet des VI sur l'anxiété-état & $\begin{array}{l}\text { Stati } \\
\text { stique }\end{array}$ & $\begin{array}{l}\text { Proba } \\
\text { bilité }\end{array}$ \\
\hline Effet du sexe & $t_{48}=$ & $\begin{array}{r}p= \\
0.25(\mathrm{NS})\end{array}$ \\
\hline Effet de la profession & $t_{48}=$ & $\begin{array}{r}p= \\
0.84(\mathrm{NS})\end{array}$ \\
\hline Effet de l'âge & $\begin{array}{r}F \\
(1,47)=2,40\end{array}$ & $\begin{array}{r}p \\
0.12(\mathrm{NS})\end{array}$ \\
\hline Effet de l'ancienneté & $\begin{array}{l}t_{48}= \\
0,61\end{array}$ & $\begin{array}{r}p= \\
0.54(\mathrm{NS})\end{array}$ \\
\hline $\begin{array}{l}\text { Effet du temps d'implication avec les personnes } \\
\text { détenues }\end{array}$ & $t_{48}=$ & $\begin{array}{r}p= \\
0.23(\mathrm{NS})\end{array}$ \\
\hline Effet du type d'établissement & $t_{48}=$ & $\begin{array}{r}p= \\
0.17(\mathrm{NS})\end{array}$ \\
\hline
\end{tabular}


Tableau $n^{\circ} 3$ : Moyennes des fréquences de copings.

\begin{tabular}{|c|c|c|c|}
\hline Mode de coping & $\begin{array}{r}\text { M } \\
\text { oyenne }\end{array}$ & Type & Ecart \\
\hline $\begin{array}{l}\text { Résolution de } \\
\text { problème }\end{array}$ & 3.5 & & 0.621 \\
\hline $\begin{array}{l}\text { Acceptation } \mathrm{de} \\
\text { confrontation }\end{array}$ & 3.2 & & 0.520 \\
\hline Prise de distance & 3.2 & & 0.480 \\
\hline Réévaluation positive & 3.5 & & 0.503 \\
\hline $\begin{array}{cc}\text { Prise } & \mathrm{de} \\
\text { responsabilité } & \end{array}$ & 28 & & 0.418 \\
\hline Fuite - Evitement & 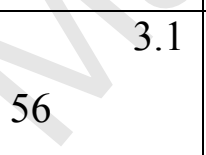 & & 0.643 \\
\hline $\begin{array}{l}\text { Recherche de soutien } \\
\text { social }\end{array}$ & 3.5 & & 0.561 \\
\hline Maîtrise de soi & 3.6 & & 0.462 \\
\hline
\end{tabular}


Figure 1: Moyennes et Ecart-type des différents modes de coping.

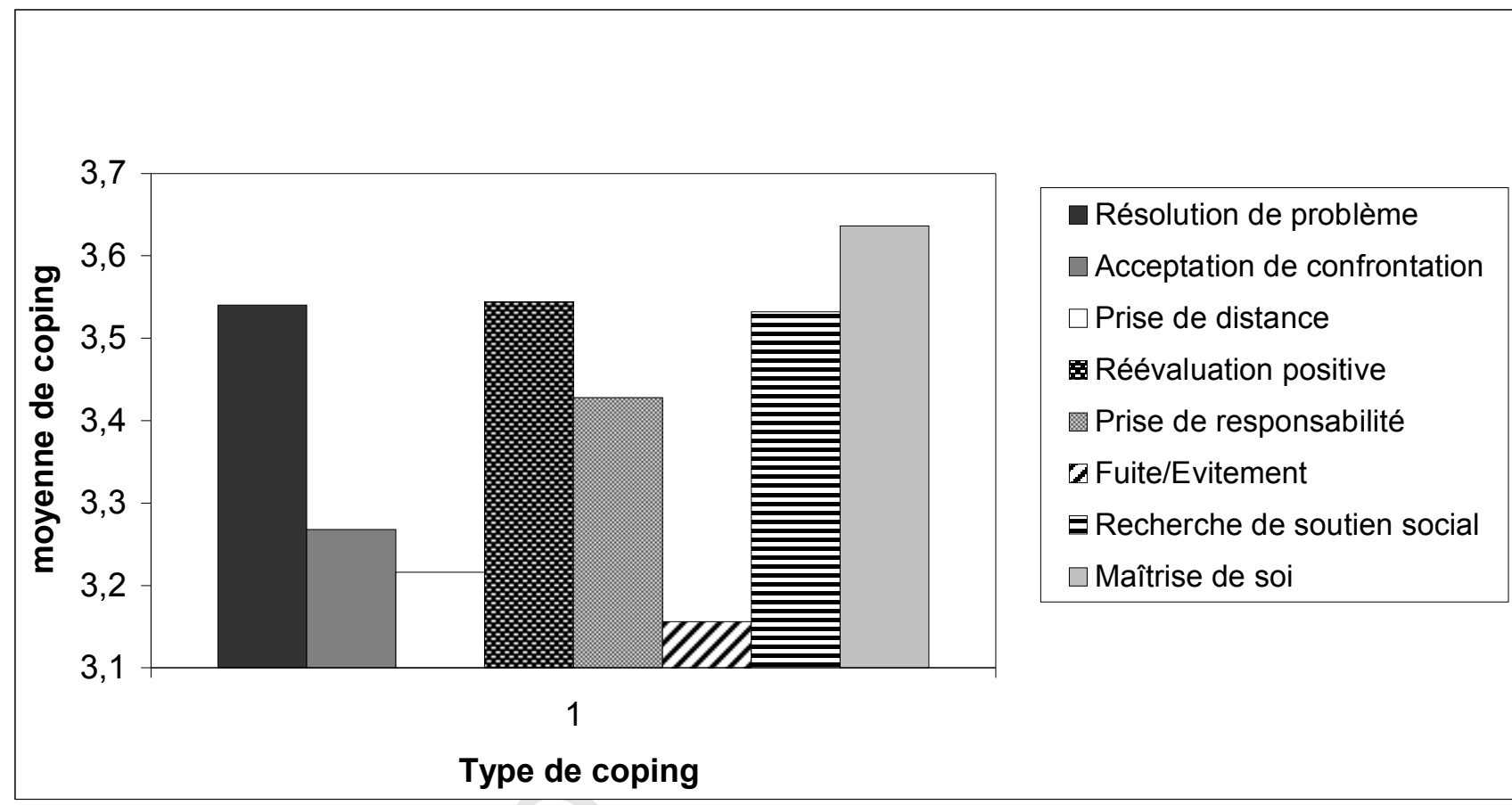


Tableau ${ }^{\circ} 4$ : Comparaison a posteriori des copings.

\begin{tabular}{|c|c|c|}
\hline Comparaisons post hoc: & tistique & $p$ \\
\hline $\begin{array}{l}\text { Résolution problème/-acceptation } \\
\text { confrontation }\end{array}$ & 72 & 2.00 \\
\hline blème -/prise de distance & 0,3 & $03 *$ \\
\hline roblème -/fuite évitement & 0,3 & $\begin{array}{r}< \\
.0001 *\end{array}$ \\
\hline $\begin{array}{l}\text { Résolution problème/- prise de } \\
\text { responsabilité }\end{array}$ & 12 & 2 \\
\hline $\begin{array}{l}\text { Résolution de problème -/recherche soutien } \\
\text { social }\end{array}$ & 08 & 92 \\
\hline Résolution de problème -/maîtrise de soi & 96 & $5^{.28}$ \\
\hline acceptation confrontation -/prise de distance & 52 & 2 \\
\hline $\begin{array}{l}\text { acceptation confrontation }-/ \text { réévaluation } \\
\text { positive }\end{array}$ & 76 & $3^{.00}$ \\
\hline $\begin{array}{l}\text { acceptation confrontation/- } \quad \text { prise } \\
\text { Responsabilité }\end{array}$ & 60 & $2^{.07}$ \\
\hline acceptation confrontation/- fuite évitement & 60 & $\begin{array}{r}.50 \\
4\end{array}$ \\
\hline $\begin{array}{l}\text { acceptation confrontation/- recherche } \\
\text { soutien }\end{array}$ & 64 & $3^{.00}$ \\
\hline acceptation confrontation/-Maîtrise de soi & 68 & $001^{<.0}$ \\
\hline prise de distance/- réévaluation positive & 28 & $001^{<.0}$ \\
\hline prise de distance/- prise de responsabilité & 12 & $8^{.01}$ \\
\hline
\end{tabular}




\begin{tabular}{|c|c|c|}
\hline prise de distance/-recherche soutien social & 16 & $5^{*} .00$ \\
\hline prise de distance- maîtrise de soi & 20 & $001^{<.0}$ \\
\hline $\begin{array}{l}\text { Réévaluation positive }- \text { prise } \mathrm{de} \\
\text { responsabilité }\end{array}$ & 0,1 & $6^{.19}$ \\
\hline prise de responsabilité/- Fuite évitement & 72 & $2^{0,0}$ \\
\hline $\begin{array}{l}\text { prise de responsabilité/- recherche soutien } \\
\text { social }\end{array}$ & 0,1 & $7^{.24}$ \\
\hline prise de responsabilité/maîtrise de soi & $\begin{array}{ll}0,2 \\
08 & \end{array}$ & 1 \\
\hline Fuite évitement/Recherche soutien sc & $\begin{array}{rr} & 0,3 \\
76 & \end{array}$ & $\begin{array}{l}<.0 \\
001 *\end{array}$ \\
\hline Fuite évitement/Maîtrise de soi & 0,4 & $001 *$ \\
\hline Recherche soutien social - maîtrise de soi & 04 & $7^{.24}$ \\
\hline
\end{tabular}

* significatif 